\title{
Grothendieck-Teichmüller and Batalin-Vilkovisky
}

Merkulov, Sergei ; Willwacher, Thomas

\begin{abstract}
It is proven that, for any affine supermanifold $M$ equipped with a constant odd symplectic structure, there is a universal action (up to homotopy) of the Grothendieck-Teichmüller Lie algebra $\mathfrak{g r t}_{1}$ on the set of quantum BV structures (i.e. solutions of the quantum master equation) on M.
\end{abstract}

DOI: https://doi.org/10.1007/s11005-014-0692-3

Posted at the Zurich Open Repository and Archive, University of Zurich ZORA URL: https://doi.org/10.5167/uzh-171984

Journal Article

Published Version

Originally published at:

Merkulov, Sergei; Willwacher, Thomas (2014). Grothendieck-Teichmüller and Batalin-Vilkovisky. Letters in Mathematical Physics, 104(5):625-634.

DOI: https://doi.org/10.1007/s11005-014-0692-3 


\title{
Grothendieck-Teichmüller and Batalin-Vilkovisky
}

\author{
SERGEI MERKULOV ${ }^{1,2}$ and THOMAS WILLWACHER ${ }^{3}$ \\ ${ }^{1}$ Department of Mathematics, Stockholm University, 10691 Stockholm, Sweden \\ ${ }^{2}$ Present address: Mathematics Research Unit, University of Luxembourg, Walferdange, \\ Grand Duchy of Luxembourg.e-mail: sergei.merkulov@uni.lu \\ ${ }^{3}$ Institute of Mathematics, University of Zurich, Winterthurerstrasse 190, 8057 Zurich, \\ Switzerland.e-mail: thomas.willwacher@math.uzh.ch
}

Received: 12 December 2013 / Revised: 18 March 2014 / Accepted: 18 March 2014

Published online: 9 April 2014 - (C) Springer Science+Business Media Dordrecht 2014

\begin{abstract}
It is proven that, for any affine supermanifold $M$ equipped with a constant odd symplectic structure, there is a universal action (up to homotopy) of the GrothendieckTeichmüller Lie algebra $\mathfrak{g r t} \mathfrak{t}_{1}$ on the set of quantum BV structures (i.e. solutions of the quantum master equation) on $M$.
\end{abstract}

Mathematics Subject Classification (1991). 81R99, 13D10.

Keywords. Grothendieck-Teichmüller group, Batalin-Vilkovisky algebras, master equation.

\section{Introduction}

Let $M$ be a finite dimensional affine $\mathbb{Z}$-graded manifold $M$ over a field $\mathbb{K}$ equipped with a constant degree 1 symplectic structure $\omega$. In particular, the ring of functions $\mathcal{O}_{M}$ is a Batalin-Vilkovisky algebra, with Batalin-Vilkovisky operator $\Delta$ and bracket $\{$,$\} . A degree 2$ function $S \in \mathcal{O}_{M}[[u]]$ is a solution of the quantum master equation on $M$ if ${ }^{1}$

$$
u \Delta S+\frac{1}{2}\{S, S\}=0,
$$

where $u$ is a formal variable of degree 2. In other words $S$ is a Maurer-Cartan element in the differential graded $(\mathrm{dg})$ Lie algebra $\left(\mathcal{O}_{M}[[u]][1], u \Delta,\{\},\right)$.

The Grothendieck-Teichmüller group $G R T_{1}$ is a pro-unipotent group introduced by Drinfeld in [3]; we denote its Lie algebra by $\mathfrak{g r t}_{1}$. In this paper we show the following result.

THEOREM 1.1 There is an $L_{\infty}$ action of the Lie algebra $\mathfrak{g r t}_{1}$ on the differential graded Lie algebra $\left(\mathcal{O}_{M}[[u]][1], u \Delta,\{\},\right)$ by $L_{\infty}$ derivations. In particular, it follows that there is an action of $G R T_{1}$ on the set of gauge equivalence classes of formal solutions of the quantum master equation, i.e. on gauge

\footnotetext{
${ }^{1}$ See [11] for an introduction into the geometry of the BV formalism.
} 
equivalence classes of Maurer-Cartan elements in the differential graded Lie algebra $\left(\hbar \mathcal{O}_{M}[[u]][[\hbar]][1], u \Delta,\{\},\right)$, where $\hbar$ is a formal deformation parameter of degree 0 .

Our main technical tool is a version of the Kontsevich graph complex, $\left(\mathrm{GC}_{2}[[u]]\right.$, $\left.d_{u}\right)$ which controls universal deformations of $\left(\mathcal{O}_{M}[[u]][1], u \Delta,\{\},\right)$ in the category of $L_{\infty}$ algebras. Using the main result of [13] we show in Section 2 that

$$
H^{0}\left(\mathrm{GC}_{2}[[u]], d_{u}\right) \simeq \mathfrak{g r t}_{1}
$$

and then use this isomorphism in Section 3 to prove the Main Theorem.

\subsection{SOME NOTATION}

In this paper $\mathbb{K}$ denotes a field of characteristic 0 . If $V=\oplus_{i \in \mathbb{Z}} V^{i}$ is a graded vector space over $\mathbb{K}$, then $V[k]$ stands for the graded vector space with $V[k]^{i}:=V^{i+k}$. For $v \in V^{i}$, we set $|v|:=i$. The phrase differential graded is abbreviated by dg. The $n$ fold symmetric product of a (dg) vector space $V$ is denoted by $\odot{ }^{n} V$, and the full symmetric product space by $\odot^{\bullet} \mathrm{V}$. For a finite group $G$ acting on a vector space $V$, we denote via $V^{G}$ the space of invariants with respect to the action of $G$, and by $V_{G}$ the space of coinvariants $V_{G}=V /\{g v-v \mid v \in V, g \in G\}$. As we always work over a field $\mathbb{K}$ of characteristic zero, we have a canonical isomorphism $V_{G} \cong V^{G}$.

We use freely the language of operads. For a background on operads we refer to the textbook [10]. For an operad $\mathcal{P}$ we denote by $\mathcal{P}\{k\}$ the unique operad which has the following property: for any graded vector space $V$ there is a one-to-one correspondence between representations of $\mathcal{P}\{k\}$ in $V$ and representations of $\mathcal{P}$ in $V[-k]$; in particular, $\mathcal{E} n d_{V}\{k\}=\mathcal{E} n d_{V[k]}$.

\section{A Variant of the Kontsevich Graph Complex}

\subsection{FROM OPERADS TO LIE ALGEBRAS}

Let $\mathcal{P}=\{\mathcal{P}(n)\}_{n \geq 1}$ be an operad in the category of dg vector spaces with the partial compositions $o_{i}: \mathcal{P}(n) \otimes \mathcal{P}(m) \rightarrow \mathcal{P}(m+n-1), 1 \leq i \leq n$. Then the map

$$
[,]: \begin{aligned}
\mathrm{P} \otimes \mathrm{P} & \longrightarrow \mathrm{P} \\
(a \in \mathcal{P}(n), b \in \mathcal{P}(m)) & \longrightarrow[a, b]:=\sum_{i=1}^{n} a \circ_{i} b-(-1)^{|a||b|} \sum_{i=1}^{m} b \circ_{i} a
\end{aligned}
$$

makes the vector space $\mathrm{P}:=\prod_{n \geq 1} \mathcal{P}(n)$ into a dg Lie algebra [4,5]. Moreover, the Lie algebra structure descends to the subspace of coinvariants $P_{\mathbb{S}}:=\prod_{n \geq 1} \mathcal{P}(n)_{\mathbb{S}_{n}}$. Via the identification of invariants and coinvariants $P_{\mathbb{S}} \cong P^{\mathbb{S}}$, we furthermore obtain a Lie algebra structure on the space of invariants $\mathrm{P}^{\mathbb{S}}:=\prod_{n \geq 1} \mathcal{P}(n)^{\mathbb{S}_{n}}$ as well. 


\subsection{AN OPERAD OF GRAPHS AND THE KONTSEVICH GRAPH COMPLEX}

For any integers $n \geq 1$ and $\geq 0$ we denote by $\mathrm{G}_{n, l}$ a set of graphs, ${ }^{2}\{\Gamma\}$, with $n$ vertices and $l$ edges such that (i) the vertices of $\Gamma$ are labelled by elements of $[n]:=$ $\{1, \ldots, n\}$, (ii) the set of edges, $E(\Gamma)$, is totally ordered up to an even permutation. For example, $\bullet \quad 2 \in \mathrm{G}_{2,1}$. The group $\mathbb{Z}_{2}$ acts freely on $\mathrm{G}_{n, l}$ for $l \geq 2$ by changes of the total ordering; its orbit is denoted by $\left\{\Gamma, \Gamma_{o p p}\right\}$. Let $\mathbb{K}\left\langle\mathrm{G}_{n, l}\right\rangle$ be the vector space over a field $\mathbb{K}$ spanned by isomorphism classes, $[\Gamma]$, of elements of $\mathrm{G}_{n, l}$ modulo the relation ${ }^{3} \Gamma_{\text {opp }}=-\Gamma$, and consider a $\mathbb{Z}$-graded $\mathbb{S}_{n}$-module,

$$
\operatorname{Gra}(n):=\bigoplus_{l=0}^{\infty} \mathbb{K}\left\langle\mathrm{G}_{n, l}\right\rangle[l] .
$$

Note that graphs with two or more edges between any fixed pair of vertices do not contribute to $\operatorname{Gra}(n)$, so that we could have assumed right from the beginning that the sets $\mathrm{G}_{n, l}$ do not contain graphs with multiple edges. The $\mathbb{S}$-module, Gra:= $\{\operatorname{Gra}(n)\}_{n \geq 1}$, is naturally an operad with the operadic compositions given by

$$
\begin{aligned}
\circ_{i}: \operatorname{Gra}(n) \otimes \operatorname{Gra}(m) & \longrightarrow \operatorname{Gra}(m+n-1) \\
\Gamma_{1} \otimes \Gamma_{2} & \longrightarrow \sum_{\Gamma \in \mathrm{G}_{\Gamma_{1}, \Gamma_{2}}^{i}}(-1)^{\sigma_{\Gamma}} \Gamma
\end{aligned}
$$

where $\mathrm{G}_{\Gamma_{1}, \Gamma_{2}}^{i}$ is the subset of $\mathrm{G}_{n+m-1, \# E\left(\Gamma_{1}\right)+\# E\left(\Gamma_{2}\right)}$ consisting of graphs, $\Gamma$, satisfying the condition: the full subgraph of $\Gamma$ spanned by the vertices labeled by the set $\{i, i+1, \ldots, i+m-1\}$ is isomorphic to $\Gamma_{2}$, and the quotient graph, $\Gamma / \Gamma_{2}$, obtained by contracting that subgraph to a single vertex, is isomorphic to $\Gamma_{1}$. The sign $(-1)^{\sigma_{\Gamma}}$ is determined by the equality

$$
\bigwedge_{e \in E(\Gamma)} e=(-1)^{\sigma_{\Gamma}} \bigwedge_{e^{\prime} \in E\left(\Gamma_{1}\right)} e^{\prime} \wedge \bigwedge_{e^{\prime \prime} \in E\left(\Gamma_{2}\right)} e^{\prime \prime} .
$$

The unique element in $\mathrm{G}_{1,0}$ serves as the unit element in the operad Gra. The associated Lie algebra of $\mathbb{S}$-invariants, $\left((\operatorname{Gra}\{-2\})^{\mathbb{S}},[],\right)$ is denoted, following notations of [13], by $\mathrm{fGC}_{2}$. Its elements can be understood as graphs from $\mathrm{G}_{n, l}$ but with labeling of vertices forgotten, e.g.

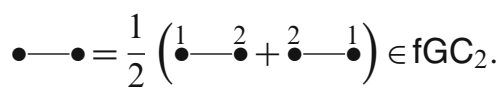

The cohomological degree of a graph with $n$ vertices and $l$ edges is $2(n-1)-l$. It is easy to check that $-\cdots$ is a Maurer-Cartan element in the Lie algebra $\mathrm{fGC}_{2}$. Hence, we obtain a dg Lie algebra

$$
\left(\mathrm{fGC}_{2},[,], d:=[\bullet-\bullet,]\right) \text {. }
$$

${ }^{2} \mathrm{~A}$ graph $\Gamma$ is, by definition, a 1-dimensional $C W$-complex whose 0 -cells are called vertices and 1-dimensional cells are called edges. The set of vertices of $\Gamma$ is denoted by $V(\Gamma)$ and the set of edges by $E(\Gamma)$.

${ }^{3}$ Abusing notations we identify from now an equivalence class $[\Gamma]$ with any of its representative $\Gamma$. 
One may define a dg Lie subalgebra, $\mathrm{GC}_{2}$, spanned by connected graphs with at least trivalent vertices and no edges beginning and ending at the same vertex. It is called the Kontsevich graph complex [7]. We leave it to the reader to verify that the subspace $\mathrm{GC}_{2}$ is indeed closed under both the differential and the Lie bracket. We refer to [13] for a detailed explanation of why studying the dg Lie subalgebra $\mathrm{GC}_{2}$ rather than full Lie algebra $\mathrm{fGC}_{2}$ should be enough for most purposes. The cohomologies of $\mathrm{GC}_{2}$ and $\mathrm{fGC}_{2}$ were partially computed in [13].

THEOREM 2.1 ([13]). (i) $H^{0}\left(\mathrm{GC}_{2}, d\right) \simeq \mathfrak{g r t}_{1}$. (ii) For any negative integer $i$, $H^{i}\left(\mathrm{GC}_{2}, d\right)=0$.

We shall introduce next a new graph complex which is responsible for the action of $G R T_{1}$ on the set of quantum master functions on an odd symplectic supermanifold.

\subsection{A VARIANT OF THE KONTSEVICH GRAPH COMPLEX}

The graph $\in \mathrm{fGC}_{2}$ has degree -1 and satisfies

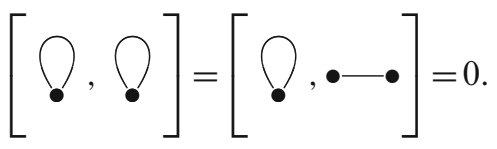

Let $u$ be a formal variable of degree 2 and consider the graph complex $\mathrm{fGC}_{2}[[u]]$ with the differential

$$
d_{u}:=d+u \Delta, \quad \text { where } \quad \Delta:=[\bigcirc,]
$$

The subspace $\mathrm{GC}_{2}[[u]] \subset \mathrm{fGC}_{2}[[u]]$ is a subcomplex of ( $\left.\mathrm{fGC}_{2}[[u]], d_{u}\right)$.

PROPOSITION 2.1. $H^{0}\left(\mathrm{GC}_{2}[[u]], d_{u}\right) \simeq \mathfrak{g r t}_{1}$ and $H^{\leq-1}\left(\mathrm{GC}_{2}[[u]]\right)=0$.

Proof. Consider a decreasing filtration of $\mathrm{GC}_{2}[[u]]$ by the powers in $u$. The first term of the associated spectral sequence is

$$
\mathcal{E}_{1}=\bigoplus_{i \in \mathbb{Z}} \mathcal{E}_{1}^{i}, \quad \mathcal{E}_{1}^{i}=\prod_{p \geq 0} H^{i-2 p}\left(\mathrm{GC}_{2}, d\right) u^{p}
$$

with the differential equal to $u \Delta$. As $H^{0}\left(\mathrm{GC}_{2}, d\right) \simeq \mathfrak{g r t}_{1}$ and $H^{\leq-1}\left(\mathrm{GC}_{2}, d\right)=0$, one gets the desired result. $H^{0}\left(\mathrm{fGC}_{2}[[u]], d_{u}\right) \simeq \mathfrak{g r t}_{1}$.

The projections $\left(\mathrm{GC}_{2}[[u]], d_{u}\right) \rightarrow\left(\mathrm{GC}_{2}, d\right)$ and $\left(\mathrm{fGC}_{2}[[u]], d_{u}\right) \rightarrow\left(\mathrm{fGC}_{2}, d\right)$ sending $u$ to 0 are maps of Lie algebras and induce isomorphisms in degree 0 cohomology. Since the isomorphisms of Theorem 2.1 (i) are maps of Lie algebras as shown in [13], so are the maps in the above Proposition. 
Remark 2.2. Let $\sigma$ be an element of $\mathfrak{g r t}_{1}$ and let $\Gamma_{\sigma}^{(0)}$ be any cycle representing the cohomology class $\sigma$ in the graph complex $\left(\mathrm{GC}_{2}, d\right)$. Then one can construct a cocycle,

$$
\Gamma_{\sigma}^{u}=\Gamma_{\sigma}^{(0)}+\Gamma_{\sigma}^{(1)} u+\Gamma_{\sigma}^{(2)} u^{2}+\Gamma_{\sigma}^{(3)} u^{3}+\ldots,
$$

representing the cohomology class $\sigma \in \mathfrak{g r t}_{1}$ in the complex $\left(\mathrm{GC}_{2}[[u]], d_{u}\right)$ by the following induction:

1st step: As $d \Gamma_{\sigma}^{(0)}=0$, we have $d\left(\Delta \Gamma_{\sigma}^{(0)}\right)=0$. As $H^{-1}\left(\mathrm{GC}_{2}, d\right)=0$, there exists $\Gamma_{\sigma}^{(1)}$ of degree -2 such that $\Delta \Gamma_{\sigma}^{(0)}=-d \Gamma_{\sigma}^{(1)}$ and hence

$$
(d+u \Delta)\left(\Gamma_{\sigma}^{(0)}+\Gamma_{\sigma}^{(1)} u\right)=0 \bmod O\left(u^{2}\right) .
$$

$n$-th step: Assume we have constructed a polynomial $\sum_{i=1}^{n} \Gamma_{\sigma}^{(i)} u^{i}$ such that

$$
(d+u \Delta) \sum_{i=1}^{n} \Gamma_{\sigma}^{(i)} u^{i}=0 \bmod O\left(u^{n+1}\right) .
$$

Then $d\left(\Delta \Gamma_{\sigma}^{(n)}\right)=0$, and, as $H^{-2 n-1}\left(\mathrm{GC}_{2}, d\right)=0$, there exists a graph $\Gamma_{\sigma}^{(n+1)}$ in $\mathrm{GC}_{2}$ of degree $-2 n-2$ such that $\left.\Delta \Gamma_{\sigma}^{(n)}\right)=-d \Gamma_{\sigma}^{(n+1)}$. Hence, $(d+u \Delta) \sum_{i=1}^{n+1} \Gamma_{\sigma}^{(i)} u^{i}=$ $0 \bmod O\left(u^{n+2}\right)$.

\section{Quantum BV Structures on Odd Symplectic Manifolds}

\subsection{MAURER-CARTAN ELEMENTS AND GAUGE TRANSFORMATIONS}

Let $\left(\mathfrak{g}=\oplus_{i \in \mathbb{Z}} \mathfrak{g}^{i},[], d,\right)$ be a dg Lie algebra and consider the dg Lie algebra $\mathfrak{g}_{\hbar}:=\hbar \mathfrak{g}[[\hbar]]=: \oplus_{i \in \mathbb{Z}} \mathfrak{g}_{\hbar}^{i}$, where $\hbar$ is a formal deformation parameter. The group $G:=\exp \left(\mathfrak{g}_{\hbar}^{0}\right)$ (which is, as a set, $\mathfrak{g}_{\hbar}^{0}$ equipped with the standard Baker-CampbellHausdorff multiplication) acts on $\mathfrak{g}_{\hbar}^{1}$,

$$
\gamma \rightarrow \exp (h) \cdot \gamma:=\mathrm{e}^{\operatorname{ad}_{h}} \gamma-\frac{\mathrm{e}^{\mathrm{ad}_{h}}-1}{\operatorname{ad}_{h}} \mathrm{~d} h,
$$

preserving its subset of Maurer-Cartan elements

$$
\mathcal{M C}\left(\mathfrak{g}_{\hbar}\right)=\left\{\gamma \in \mathfrak{g}_{\hbar}^{1} \mid d \gamma+\frac{1}{2}[\gamma, \gamma]=0\right\} .
$$

We call the $G$-orbits in $\mathcal{M C}\left(\mathfrak{g}_{\hbar}\right)$ the gauge equivalence classes of Maurer-Cartan elements.

The group of $L_{\infty}$ automorphism of $\mathfrak{g}$ acts on $\mathcal{M C}\left(\mathfrak{g}_{\hbar}\right)$ by the formula

$$
F \cdot \gamma:=\sum_{n \geq 1} \frac{1}{n !} F_{n}(\gamma, \ldots, \gamma)
$$


where $F_{n}$ is the $n$-th component of the $L_{\infty}$ morphism. In particular, let $f$ be an $L_{\infty}$ derivation of $\mathfrak{g}$ without linear term. It exponentiates to an $L_{\infty}$ automorphism $\exp (f)$ of $\mathfrak{g}$, which acts on $\mathcal{M C}\left(\mathfrak{g}_{\hbar}\right)$, and in particular on the set of gauge equivalence classes. By a small calculation one may check that if we change $f$ by homotopy, i.e. by adding $\mathrm{d} h$ for some degree 0 element $h$ of the Chevalley-Eilenberg complex of $\mathfrak{g}$, then the induced actions of $\exp (f)$ and $\exp (f+\mathrm{d} h)$ on the set of gauge equivalence classes agree.

\subsection{QUANTUM BV MANIFOLDS}

Let $M$ be a $\mathbb{Z}$-graded manifold equipped with an odd symplectic structure $\omega$ (of degree 1). There always exist so-called Darboux coordinates, $\left(x^{a}, \psi_{a}\right)_{1 \leq a \leq n}$, on $M$ such that $\left|\psi_{a}\right|=-\left|x^{a}\right|+1$ and $\omega=\sum_{a} \mathrm{~d} x^{a} \wedge \mathrm{d} \psi_{a}$. The odd symplectic structure makes, in the obvious way, the structure sheaf into a Lie algebra with brackets, $\{$,$\} , of degree -1$. A less obvious fact is that $\omega$ induces a degree -1 differential operator, $\Delta_{\omega}$, on the invertible sheaf of semidensities, $\operatorname{Ber}(M)^{\frac{1}{2}}$ [6]. Any choice of a Darboux coordinate system on $M$ defines an associated trivialization of the sheaf $\operatorname{Ber}(M)^{\frac{1}{2}}$; if one denotes the associated basis section of $\operatorname{Ber}(M)^{\frac{1}{2}}$ by $D_{x, \psi}$, then any semidensity $D$ is of the form $f(x, \psi) D_{x, \psi}$ for some smooth function $f(x, \psi)$, and the operator $\Delta_{\omega}$ is given by

$$
\Delta_{\omega}\left(f(x, \psi) D_{x, \psi}\right)=\sum_{a=1}^{n} \frac{\partial^{2} f}{\partial x^{a} \partial \psi_{a}} D_{x, \psi} .
$$

Let $u$ be a formal parameter of degree 2. A quantum master function on $M$ is a $u$-dependent semidensity $D$ which satisfies the equation

$$
\Delta_{\omega} D=0
$$

and which admits, in some Darboux coordinate system, a form

$$
D=\mathrm{e}^{\frac{S}{u}} D_{x, \psi},
$$

for some $S \in \mathcal{O}_{M}[[u]]$ of total degree 2, where $\mathcal{O}_{M}$ is the algebra of functions on $M$. In the literature it is this formal power series in $u$ which is often called a quantum master function. Let us denote the set of all quantum master functions on $M$ by $\mathcal{Q M}(M)$. It is easy to check that the equation $\Delta_{\omega} D=0$ is equivalent to the following one,

$$
u \Delta S+\frac{1}{2}\{S, S\}=0,
$$

where $\Delta:=\sum_{a=1}^{n} \frac{\partial^{2}}{\partial x^{a} \partial \psi_{a}}$. This equation is often called the quantum master equation, while a triple $(M, \omega, S \in \mathcal{Q M}(M))$ a quantum $B V$ manifold. 
Let us assume from now on that $M$ is affine or formal (i.e., we work with $\infty$-jets of functions at some point) and that a particular Darboux coordinate system is fixed on $M$ up to affine transformations ${ }^{4}$ so that the algebra of function on $M$ is $\mathcal{O}_{M} \cong \mathbb{K}\left[x^{a}, \psi_{a}\right]$ or $\mathcal{O}_{M} \cong \mathbb{K}\left[\left[x^{a}, \psi_{a}\right]\right]$.

For later reference we will also consider solutions of (2) that depend on a formal deformation parameter $\hbar$ of degree $0, S \in \hbar \mathcal{O}_{M}[[u]][[\hbar]]$. We will call the set of such $S$ the set of formal solutions of the quantum master equation and denote it by $\mathcal{Q M}_{\hbar}(M)$.

\subsection{AN ACTION OF GRT $T_{1}$ ON QUANTUM MASTER FUNCTIONS}

The constant odd symplectic structure on $M$ makes $\mathcal{O}_{M}$ into a representation

$$
\begin{aligned}
\rho: \operatorname{Gra}(n) & \longrightarrow \operatorname{End}_{V}(n)=\operatorname{Hom}_{\text {cont }}\left(\mathcal{O}_{M}^{\otimes n}, \mathcal{O}_{M}\right) \\
\Gamma & \longrightarrow \Phi_{\Gamma}
\end{aligned}
$$

of the operad Gra as follows:

$$
\begin{aligned}
& \Phi_{\Gamma}\left(S_{1}, \ldots, S_{n}\right) \\
& \quad:=\pi\left(\prod_{e \in E(\Gamma)} \Delta_{e}\left(S_{1}\left(x_{(1)}, \psi_{(1)}, u\right) \otimes S_{2}\left(x_{(2)}, \psi_{(2)}, u\right) \otimes \ldots \otimes S_{n}\left(x_{(n)}, \psi_{(n)}, u\right)\right)\right)
\end{aligned}
$$

where, for an edge $e$ connecting vertices labeled by integers $i$ and $j$,

$$
\Delta_{e}=\sum_{a=1}^{n} \frac{\partial}{\partial x_{(i)}^{a}} \frac{\partial}{\partial \psi_{a(j)}}+\frac{\partial}{\partial \psi_{a(i)}} \frac{\partial}{\partial x_{(j)}^{a}}
$$

with the subscript $(i)$ or $(j)$ indicating that the derivative operator is to be applied to the $i$-th of $j$-th factor in the tensor product. The symbol $\pi$ in (4) denotes the multiplication map,

$$
\pi: \quad V^{\otimes n} \longrightarrow V
$$

Let $V:=\mathcal{O}_{M}[[u]]$. Then by $u$-linear extension, we obtain a continuous representation (in the category of topological $\mathbb{K}[[u]]$-modules)

$$
\operatorname{Gra}[[u]] \longrightarrow \operatorname{End}_{V}=\operatorname{Hom}_{\text {cont }}\left(V^{\otimes \cdot}, V\right) .
$$

The space $V[1]$ is a topological dg Lie algebra with differential $u \Delta$ and Lie bracket $\{$,$\} . These data define a Maurer-Cartan element, \gamma_{\mathcal{Q M}}:=u \Delta \oplus\{$,$\} in the$

\footnotetext{
${ }^{4}$ This is not a serious loss of generality as any quantum master equation can be represented in the form (2). Our action of $G R T_{1}$ on $\mathcal{Q} \mathcal{M}_{\hbar}(M)$ depends on the choice of an affine structure on $M$ in exactly the same way as the classical Kontsevich's formula for a universal formality map [8] depends on such a choice. A choice of an appropriate affine connection on $M$ and methods of the paper [2] can make our formulae for the $G R T_{1}$ action invariant under the group of symplectomorphisms of $(M, \omega)$; we do not address this globalization issue in the present note.
} 
Lie algebra $\left(\operatorname{End}_{V}\{-2\}\right)^{\mathbb{S}} \subset C E^{\bullet}(V, V)$, where $C E^{\bullet}(V, V)$ is the Lie algebra of coderivations

$$
\begin{aligned}
C E^{\bullet}(V, V) & =\left(\operatorname{Coder}\left(\odot^{\bullet \geq 1}(V[2])\right),[,]\right) \text { with } \\
C E^{\bullet}(V, V)_{(m)} & :=\operatorname{Hom}\left(\odot^{\bullet \geq m+1}(V[2]), V[2]\right),
\end{aligned}
$$

of the standard graded co-commutative coalgebra, $\odot^{\bullet} \geq 1(V[2])$, co-generated by a vector space $V$. The set $\mathcal{M C}\left(C E^{\bullet}(V, V)\right)$ can be identified with the set of $L_{\infty}$ structures on the space $V[1]$.

The map sending an operad $\mathcal{P}$ to the Lie algebra of invariants $\prod_{n} \mathcal{P}\{-2\}(n)^{\mathbb{S}_{n}}$ is functorial. Hence, from the representation (4) we obtain a map of graded Lie algebras

$$
\mathrm{fGC}_{2}[[u]] \cong(\operatorname{Gra}\{-2\}[[u]])^{\mathbb{S}} \rightarrow\left(\operatorname{End}_{V}\{-2\}\right)^{\mathbb{S}} \subset C E^{\bullet}(V, V)
$$

One checks that the Maurer-Cartan element

$$
\bullet-\bullet+u \in \mathrm{fGC}_{2}[[u]]
$$

is sent to the Maurer-Cartan element $\gamma_{\mathcal{Q M}} \in C E^{\bullet}(V, V)$. Hence, we obtain a morphism of dg Lie algebras

$$
\left(\mathrm{fGC}_{2}[[u]],[,], d_{h}\right) \longrightarrow\left(C E^{\bullet}(V, V),[,], \delta:=\left[\gamma_{\mathcal{Q M}},\right]\right),
$$

and by restriction a morphism

$$
\Phi:\left(\mathrm{GC}_{2}[[u]],[,], d_{h}\right) \longrightarrow\left(C E^{\bullet}(V, V),[,], \delta:=\left[\gamma_{\mathcal{Q M}},\right]\right),
$$

Hence, we also obtain a morphism of their cohomology groups,

$$
\mathfrak{g r t}_{1} \simeq H^{0}\left(\mathrm{GC}_{2}[[u]], d_{u}\right) \longrightarrow H^{0}\left(C E^{\bullet}(V, V), \delta\right) .
$$

Let $\sigma$ be an arbitrary element in $\mathfrak{g r t}_{1}$ and let $\Gamma_{\sigma}^{u}$ be a cocycle representing $\sigma$ in the graph complex $\left(\mathrm{GC}_{2}[[u]], d_{u}\right)$. We may assume that $\Gamma_{\sigma}^{u}$ consists of graphs with at least 4 vertices; see [13]. Then the element $\Phi\left(\Gamma_{\sigma}^{u}\right)$ describes an $L_{\infty}$ derivation of the Lie algebra $V[1]$ without the linear term. By exponentiation we obtain an $L_{\infty}$ automorphism,

$$
F^{\sigma}=\left\{F_{n}^{\sigma}: \odot^{n} V \longrightarrow V[2-2 n]\right\}_{n \geq 1},
$$

of the dg Lie algebra $(V[1], u \Delta,\{\}$,$) with F_{1}^{\sigma}=$ Id. Hence, for any formal quantum master function $S \in \mathcal{Q M}_{\hbar}(M)$ the series

$$
S^{\sigma}:=S+\sum_{n \geq 2} \frac{1}{n !} F_{n}^{\sigma}(S, \ldots, S)
$$


gives again a formal quantum master function. ${ }^{5}$ The induced action on gauge equivalence classes of such functions is well defined, i.e. it does not depend on the representative $\Gamma_{\sigma}^{u}$ chosen. This is the acclaimed homotopy action of $G R T_{1}$ on $\mathcal{Q M}_{\hbar}(M)$ for any affine odd symplectic manifold $M$.

Remark 3.1. As pointed out by one of the referees, there is also a stronger notion of "homotopy action" that holds in our setting. We will only consider the infinitesimal version. Then, we do not only have a Lie algebra morphism $\mathfrak{g r t}_{1} \rightarrow$ $H^{0}\left(C E^{\bullet}(V, V)\right)$, but an $L_{\infty}$ morphism $\mathfrak{g r t}_{1} \rightarrow C E^{\bullet}(V, V)$ as follows. First, consider the truncated version $\left(\mathrm{GC}_{2}[[u]]\right)^{t r}$ of the dg Lie algebra $\mathrm{GC}_{2}[[u]]$, which is by definition the same as $\mathrm{GC}_{2}[[u]]$ in negative degrees, zero in positive degrees, and consists of the degree zero cocycles in degree zero. By Proposition 2.1 the canonical projection $\left(\mathrm{GC}_{2}[[u]]\right)^{t r} \rightarrow \mathfrak{g r t}_{1}$ is a quasi-isomorphism. Hence we can obtain the desired $L_{\infty}$ morphism $\mathfrak{g r t}_{1} \rightarrow C E^{\bullet}(V, V)$ by lifting the zig-zag

$$
\mathfrak{g r t}_{1} \stackrel{\sim}{\longleftarrow}\left(\mathrm{GC}_{2}[[u]]\right)^{t r} \longrightarrow C E^{\bullet}(V, V)
$$

This proves the first claim of the main Theorem.

Remark 3.2. It is a well-known result due to Tamarkin [12] that the Grothendieck Teichmüller group $G R T_{1}$ acts on the operad of chains of the little disks operad. In fact, one can show that this $G R T_{1}$ action extends to an action on the operad of chains of the framed little disks operad, which is quasi-isomorphic to the BatalinVilkovisky operad. Hence, one obtains in particular an action of $G R T_{1}$ on the set of Batalin-Vilkovisky algebra structures on any vector space, and on their deformations, up to homotopy. In our setting the algebra $\mathcal{O}_{M}$ is an algebra over the framed little disks operad. Any solution $S=S_{0}+u S_{1}+u^{2} S_{2}+\cdots$ of the master equation (2) yields a deformation of the Batalin-Vilkovisky structure on $\mathcal{O}_{M}$, up to homotopy. Concretely, to $S$ one may associate a $B V_{\infty}^{c o m}$-structure (see [9] or [1, section 5.3]), whose $n$-th order "BV" operator is defined as $\Delta_{n}:=\left[S_{n}, \cdot\right]$ (notation as in [1, section 5.3]). The $G R T_{1}$ action on solutions of the master equation described above can hence be seen as a shadow of this more general action of $G R T_{1}$ on the framed little disks operad. However, we leave the details to elsewhere.

\section{Acknowledgements}

We are grateful to $\mathrm{K}$. Costello and to the anonymous referees for useful critical comments.

${ }^{5}$ The series trivially converges since we work in the formal setting, i.e. $S=\hbar(\cdots)$. Ideally, of course, one hopes to have a nonzero convergence radius in $\hbar$, but we cannot guarantee this. 


\section{References}

1. Campos, R., Merkulov, S., Willwacher, T.: The Frobenius properad is Koszul (preprint arXiv:1402.4048)

2. Dolgushev, V.: Covariant and equivariant formality theorems. Adv. Math. 191(1), 147177 (2005)

3. Drinfeld, V.: On quasitriangular quasi-Hopf algebras and a group closely connected with $\operatorname{Gal}(\bar{Q} / Q)$. Leningrad Math. J. 2(4), 829-860 (1991)

4. Gerstenhaber, M., Voronov, A.A.: Homotopy $G$-algebras and moduli space operad. IMRN 3, 141-153 (1995)

5. Kapranov, M., Manin, Yu.I.: Modules and Morita theorem for operads. Am. J. Math. 123(5), 811-838 (2001)

6. Khudaverdian, H.: Semidensities on odd symplectic supermanifolds. Commun. Math. Phys. 247, 353-390 (2004)

7. Kontsevich, M.: Formality conjecture. In: Sternheimer, D., et al. (eds.) Deformation Theory and Symplectic Geometry, pp. 139-156. Kluwer, Dordrecht (1997)

8. Kontsevich, M.: Deformation quantization of Poisson manifolds. Lett. Math. Phys. 66, 157-216 (2003)

9. Kravchenko, O.: Deformations of Batalin-Vilkovisky algebras. In: Poisson Geometry (Warsaw, 1998), Banach Center Publ., vol. 51, pp. 131-139. Polish Acad. Sci., Warsaw (2000)

10. Loday, J.-L., Vallette, B.: Algebraic Operads. Number 346 in Grundlehren der mathematischen Wissenschaften. Springer, Berlin (2012)

11. Schwarz, A.: Geometry of Batalin-Vilkovisky quantization. Commun. Math. Phys. 155, 249-260 (1993)

12. Tamarkin, D.: Action of the Grothendieck-Teichmüller group on the operad of Gerstenhaber algebras (preprint arXiv:math/0202039)

13. Willwacher, T.: M. Kontsevich's graph complex and the Grothendieck-Teichmüller Lie algebra (preprint arXiv:1009.1654) 